\title{
Improvements in cognition, mood and behaviour following commencement of continuous subcutaneous insulin infusion therapy in children with type 1 diabetes mellitus: a pilot study
}

\author{
S. Knight • E. Northam • S. Donath • A. Gardner • \\ N. Harkin • C. Taplin • P. Joy • F. J. Cameron • \\ G. R. Ambler
}

Received: 22 July 2008 / Accepted: 7 October 2008 / Published online: 6 November 2008

(C) Springer-Verlag 2008

\begin{abstract}
Aims/hypothesis Anecdotally, parents and teachers of children with type 1 diabetes mellitus report improvements in behaviour and learning following the commencement of continuous subcutaneous insulin infusion (CSII). This study aimed to investigate changes in cognition, mood and behaviour following commencement of CSII in children with type 1 diabetes.

Methods Children $(n=32)$ with type 1 diabetes aged 6-16 years and starting CSII at two Australian centres underwent behavioural, cognitive and glycaemic assessments prior to the commencement of CSII and 6-8 weeks after its
\end{abstract}

\author{
S. Knight $\cdot$ E. Northam $\cdot$ S. Donath $\cdot$ F. J. Cameron \\ Murdoch Children's Research Institute, \\ Melbourne, VIC, Australia \\ S. Knight $\cdot$ E. Northam \\ School of Behavioural Science, \\ University of Melbourne, \\ Melbourne, VIC, Australia \\ E. Northam $\cdot$ F. J. Cameron \\ Royal Children's Hospital, \\ Melbourne, VIC, Australia \\ A. Gardner $\cdot$ N. Harkin • C. Taplin • P. Joy • G. R. Ambler $(\triangle)$ \\ Institute of Endocrinology and Diabetes, \\ The Children's Hospital at Westmead, \\ Locked Bag 4001, \\ Westmead, NSW 2145, Australia \\ e-mail: geoffa@chw.edu.au \\ G. R. Ambler \\ Discipline of Paediatrics and Child Health, \\ University of Sydney, \\ Sydney, NSW, Australia
}

start. A comprehensive cognitive test battery was administered, comprising measures of intelligence, attention, processing speed and executive skills. Behaviour and mood were assessed using the Behaviour Assessment System for Children-Second Edition. Continuous glucose monitoring was performed over a $72 \mathrm{~h}$ period and $\mathrm{HbA}_{1 \mathrm{c}}$ was measured at both time-points.

Results After commencement of CSII, there were significant improvements in $\mathrm{HbA}_{1 \mathrm{c}}$, a reduction in hyperglycaemia and blood glucose variation and an increase in normoglycaemia. Significant improvements were observed in perceptual reasoning, selective attention, divided attention, cognitive flexibility and working memory. Fewer mood-related symptoms were reported (parent, teacher and self-report) and fewer behavioural problems (parent reports)

Conclusions/interpretation In this uncontrolled pilot study, children with type 1 diabetes demonstrated significant improvements in measures of metabolic control, mood and behaviour and in some complex cognitive skills after commencing CSII therapy.

Keywords Behaviour - Blood glucose levels · Cognition . Haemoglobin $\mathrm{A}_{1 \mathrm{c}} \cdot$ Mood . Type 1 diabetes
Abbreviations
BASC-2 Behaviour Assessment System for Children- Second Edition
BGL blood glucose level
CGMS continuous glucose monitoring system
CONGA continuous overlapping net glycaemic action
CSII continuous subcutaneous insulin infusion
SES socioeconomic status 


\section{Introduction}

There is now a substantial body of literature indicating that children with type 1 diabetes are at increased risk of cognitive and behavioural difficulties [1-3]. Both intercurrent fluctuations in blood glucose level (BGL) $[4,5]$ and longer-term metabolic control [6] have been shown to affect cognition and behaviour. The cognitive skills that appear most affected by fluctuating BGLs include attention, working memory, complex information processing and cognitive flexibility $[2,7,8]$.

Data supporting the benefits of insulin delivery via continuous subcutaneous insulin infusion (CSII) in children with type 1 diabetes are increasing, with studies showing improved metabolic control, lower rates of hypoglycaemia and improved quality of life [9-13]. Anecdotally, families and teachers frequently report improvements in cognition, mood and behaviour after the commencement of insulin pump therapy, although to date there have been no published attempts to study this systematically.

The purpose of this uncontrolled pilot study was to investigate whether improved metabolic control associated with commencing CSII is associated with measurable improvements in cognition, mood and behaviour.

\section{Methods}

Study design Children with pre-existing type 1 diabetes attending The Children's Hospital Westmead, Sydney $(n=15)$ or The Royal Children's Hospital, Melbourne $(n=17)$, aged 6-16 years, were recruited as they were started on CSII. This age range corresponded to the validated age range for the selected behavioural and cognitive tests employed. The children commenced CSII for a range of reasons including unstable glycaemia, a desire to improve control, lifestyle flexibility and personal or physician therapeutic preference. The decision to commence CSII was independent of any involvement in the study; there were no other specific eligibility criteria. Ethical approval was obtained from the Human Ethics Research Committees at both participating hospitals and informed consent was obtained from participants and parents. Psychometric and glycaemic assessments were conducted in the week before CSII was commenced and 6-8 weeks after CSII commencement.

Socioeconomic status (SES) was assessed based on the principal income earner of the family, and quantified using the Australian and New Zealand Standard Classification of Occupations [14], which provides a 1-8 rating scale (high SES 1-2.5, medium SES 2.6-5.4, low SES 5.5-8).

The Behaviour Assessment System for Children-Second Edition (BASC-2) This standardised instrument was used to assess mood and behaviour using parent and teacher report forms. Self-report forms were also completed by children aged 9 years and over. Analyses of BASC-2 data were carried out using BASC-2-enhanced ASSIST, which generates summary T scores (mean of 50 and SD of 10) standardised for age and sex for externalising and internalising behaviour. The externalising scale measures behaviour and includes symptoms of hyperactivity, aggression and conduct problems, while the internalising scale measures mood and comprises anxiety, depression and somatisation scores. High scores indicate a greater tendency to psychopathology.

Cognitive test battery The instruments used in this study are described in Table 1. Test selection was guided by previous research indicating that complex information processing tasks, vigilance, efficient decision making, processing speed and working memory are the skills most sensitive to intercurrent or recent blood glucose variations and to longer-term diabetes-related effects $[1,2,7,8]$. The selected neuropsychological tests are accepted as valid measures of the variables of interest $[15,16]$ and are of sufficient task difficulty to be sensitive to subtle changes in cognitive functioning. In order to minimise practise effects, the follow-up assessment protocol included alternative forms where possible, as shown in Table 1 . Some testretest data are available from the testing manuals $[17,18]$ and are compared with our results.

Cognitive assessment was performed only if the child had a BGL between 4 and $18 \mathrm{mmol} / \mathrm{l}$, based on limited available data suggesting that acute effects on cognition are unlikely inside this range [19-21].

Table 1 Cognitive test battery

\begin{tabular}{ll}
\hline Construct & Measure \\
\hline $\begin{array}{l}\text { General intelligence } \\
\text { Vocabulary }\end{array}$ & Vocabulary \\
Perceptual reasoning & Block design (WISC-IV). \\
& Alternative version from WASI \\
Immediate attention span & Digit span subtest (WISC-IV). \\
& Alternative version from CMS \\
Selective attention & Sky search (TEA-Ch) ${ }^{\mathrm{a}, \mathrm{b}}$ \\
Divided attention & Score DT (TEA-Ch) \\
Sustained attention & Score (TEA-Ch) \\
Cognitive flexibility & Contingency naming test ${ }^{\mathrm{a}}$ \\
Concept formation & Verbal fluency test (DKEFS) \\
Organisation & Rey complex figure \\
Working memory & Letter number sequencing (WISC-IV) \\
New learning & Paired associative learning (Nott) \\
\hline
\end{tabular}

${ }^{\mathrm{a}}$ A number of tests were timed to provide a measure of processing speed

${ }^{\mathrm{b}}$ Alternative version available within same test instrument

CMS, Children's memory scale; DKEFS, Delis-Kaplan Executive Function System; TEA-Ch, Test of Everyday Attention for Children; WASI, Wechsler Abbreviated Scale of Intelligence; WISC-IV, Wechsler Intelligence Scale for Children-Fourth Edition 
Glycaemic assessment A continuous glucose monitoring system (CGMS) (Medtronic CGMS System Gold; Medtronic, Northridge, CA, USA) was used by participants for a $72 \mathrm{~h}$ period in the 7 days prior to CSII commencement and at a time-point 6-8 h afterwards. Data were summarised using CGMS Gold software and an algorithm previously reported by this group [22], which examines a number of variables of BGL variation including mean of daily differences and continuous overlapping net glycaemic action (CONGA). CONGA is a measure of intra-day glycaemic variation over specified time periods of 1,2 or $4 \mathrm{~h}$ with reported mean values of CONGA1, CONGA2 and CONGA4 being 0.7, 0.8 and 1.0, respectively, in non-diabetic individuals and $2.5,3.6$ and 4.6 in randomly selected diabetic patients [22]. The percentage of time spent in hypoglycaemic (CGMS $<4 \mathrm{mmol} / \mathrm{l}$ ), normoglycaemic (4-12 mmol/l) and hyperglycaemic $(>12 \mathrm{mmol} / \mathrm{l})$ glucose ranges was calculated for each participant.

$\mathrm{HbA}_{1 \mathrm{c}}$ was measured by capillary blood sampling at the commencement of CSII and at the second assessment 6-8 weeks later. Measurement was by HPLC (Variant; Bio-Rad Laboratories, Hercules, USA, USA) with a nondiabetic range of $4.6-6.5 \%$.

Statistical analyses Paired-sample $t$ tests or Wilcoxon signed-ranks test (for non-normal data) were used to analyse change in variables at baseline and follow-up. Relationships between variables were explored by regression analysis. For cognitive and behavioural variables, standardised scores are shown where possible; otherwise raw scores. In order to indicate size of change, $z$ scores are shown for each test measure.

\section{Results}

Thirty-two children (57\% male) participated in the study with mean age 12.9 years (range 6.2-15.9 years). Mean duration of diabetes was 3.25 years $(0.24-12.7$ years). The median SES score of 3 was in the higher-status end of the medium range with 19\% low SES, 31\% medium SES and $50 \%$ high SES.

Cognition As shown in Table 2, a significantly better performance was observed after the commencement of CSII on the following tasks: perceptual reasoning, selective attention, divided attention, cognitive flexibility (trials 2, 3 and 4) and working memory. The change we found in perceptual reasoning score of +1.13 was approximately double the test-retest effect of 0.6 reported in the test manual [17] and similarly the change we found in working

Table 2 Cognitive variables at baseline and follow-up

\begin{tabular}{|c|c|c|c|c|}
\hline Measure & Baseline & Follow-up & Change in $z$ score & $p$ value \\
\hline \multicolumn{5}{|l|}{ General intelligence } \\
\hline Vocabulary & $10.53 \pm 2.93$ & $10.94 \pm 2.72$ & 0.14 & 0.17 \\
\hline Perceptual reasoning & $10.50 \pm 3.16$ & $11.63 \pm 3.38$ & 0.38 & $<0.05$ \\
\hline \multicolumn{5}{|l|}{ Attention } \\
\hline Immediate attention & $9.97 \pm 2.99$ & $9.81 \pm 3.27$ & 0.16 & 0.71 \\
\hline Sustained attention & $9.24 \pm 3.08$ & $8.93 \pm 3.08$ & 0.31 & 0.62 \\
\hline Selective attention & $9.32 \pm 2.71$ & $10.45 \pm 2.66$ & 0.38 & $<0.05$ \\
\hline Divided attention & $9.27 \pm 2.95$ & $10.47 \pm 2.21$ & 0.40 & $<0.001$ \\
\hline \multicolumn{5}{|l|}{$\begin{array}{l}\text { Cognitive flexibility } \\
\text { (contingency namimg test) }\end{array}$} \\
\hline Trial 1 & $-0.28 \pm 1.27$ & $0.05 \pm 1.24$ & 0.33 & 0.15 \\
\hline Trial 2 & $0.04 \pm 1.44$ & $0.53 \pm 1.48$ & 0.49 & $<0.05$ \\
\hline Trial 3 & $-0.32 \pm 1.07$ & $0.94 \pm 1.58$ & 1.26 & $<0.001$ \\
\hline Trial 4 & $0.16 \pm 1.42$ & $0.79 \pm 1.19$ & 0.63 & $<0.05$ \\
\hline \multicolumn{5}{|l|}{ Planning and organisation $^{\mathrm{a}}$} \\
\hline Rey complex figure copy & $28.31 \pm 6.29$ & $27.80 \pm 6.40$ & & 0.45 \\
\hline Rey complex figure organisation & $4.50 \pm 1.11$ & $4.56 \pm 1.34$ & & 0.68 \\
\hline Working memory & $10.31 \pm 2.47$ & $11.25 \pm 2.95$ & 0.31 & $<0.05$ \\
\hline \multicolumn{5}{|l|}{ New learning ${ }^{a}$} \\
\hline Immediate recall & $26.10 \pm 3.06$ & $25.93 \pm 3.26$ & & 0.76 \\
\hline Delayed recall & $9.66 \pm 0.77$ & $9.59 \pm 0.78$ & & 0.65 \\
\hline \multicolumn{5}{|l|}{ Concept formation } \\
\hline Letter fluency & $10.10 \pm 3.16$ & $10.63 \pm 3.74$ & 0.18 & 0.19 \\
\hline Semantic fluency & $10.97 \pm 2.86$ & $9.20 \pm 2.86$ & -0.59 & $<0.005$ \\
\hline
\end{tabular}

Standardised scores are shown where available or raw scores ${ }^{\mathrm{a}}$ for baseline and follow-up 
Table 3 Comparison of mood and behaviour before and after commencement of CSII: parent, teacher and self-reports

Values are means $\pm \mathrm{SD}$

\begin{tabular}{llccc}
\hline Measure & Baseline & Follow-up & $\begin{array}{c}\text { Improvement } \\
\text { in } z \text { score }\end{array}$ & $p$ value \\
\hline Mood & & & & \\
Parent & $57.68 \pm 11.45$ & $48.06 \pm 8.05$ & 0.96 & $<0.001$ \\
Teacher & $52.83 \pm 8.91$ & $47.39 \pm 5.70$ & 0.54 & $<0.005$ \\
Self & $46.43 \pm 7.00$ & $42.57 \pm 6.04$ & 0.39 & $<0.005$ \\
Behaviour & & & & $<0.001$ \\
Parent & $52.81 \pm 8.55$ & $47.68 \pm 8.55$ & 0.51 & 0.14 \\
Teacher & $46.00 \pm 5.18$ & $45.25 \pm 4.18$ & 0.08 & 0.06 \\
Self & $49.34 \pm 8.97$ & $46.10 \pm 8.25$ & 0.32 & \\
\hline
\end{tabular}

memory of +0.94 was approximately double the test-retest change of 0.4 reported [18]. No changes in performance were observed for vocabulary, immediate and sustained attention, planning and organisation, new learning or letter fluency. Performance on a task of semantic fluency was significantly poorer at follow-up.

Mood and behaviour Data are summarised in Table 3. Children demonstrated fewer mood-related symptoms (internalising problems), based on parent $(p<0.001)$, teacher $(p<0.005)$ and self $(p<0.005)$ reports after commencement of CSII. Parent reports indicated fewer behavioural problems (externalising problems) following commencement of CSII $(p<0.001)$, while self-reports of change in behaviour approached significance $(p=0.06)$. There was no significant change based on teacher reports.

Glycaemia There was a significant improvement in $\mathrm{HbA}_{1 \mathrm{c}}$ from a mean of $8.23 \%$ to $7.48 \%(p<0.01)$ after the commencement of insulin pump therapy (Table 4). Time spent in the normoglycaemic range increased by $24 \%$ $(p<0.01)$ and time spent in the hyperglycaemic range decreased by $30 \%(p<0.05)$. There was no significant change in time spent in the hypoglycaemic range. Glycaemic variability was reduced after commencement of CSII (Table 4) as measured by CONGA1 $(p<0.05)$ and CONGA4 $(p<0.05)$ and a trend to reduction in CONGA2 $(p=0.07)$ [22].

Regression analyses did not demonstrate a direct relationship between improvement in $\mathrm{HbA}_{1 \mathrm{c}}$ or other glycaemic variables and improvement in measures of mood, behaviour or cognition.

\section{Discussion}

In this preliminary study of children commencing CSII, improvements in glycaemic control, cognition, mood and behaviour were observed over the first 6-8 weeks. These preliminary findings support anecdotal reports from families after commencing CSII therapy and limited information from the literature about the association between BGLs and cognition, mood and behaviour. We are only aware of one other small observational study in children commencing CSII therapy. Daley et al. [23] reported improved classroom concentration in four boys in on-task (20\% improvement) and off-task (34\% improvement) behaviour after insulin pump commencement, which was associated with improved stability of BGLs.
Table 4 Glycaemic variables at baseline and follow-up

Values are means $\pm \mathrm{SD}$

\begin{tabular}{lccc}
\hline Variable & Baseline & Follow-up & $p$ value \\
\hline BGL at testing $(\mathrm{mmol} / \mathrm{l})$ & $12.94 \pm 4.13$ & $11.14 \pm 4.03$ & 0.07 \\
$\mathrm{HbA}_{1 \mathrm{c}}(\%)$ & $8.23 \pm 0.90$ & $7.48 \pm 0.59$ & $<0.0001$ \\
CGMS & & & \\
Per cent of time in state of: & & & \\
Normoglycaemia $(4-12 \mathrm{mmol} / \mathrm{l})$ & $54 \pm 21$ & $67 \pm 18$ & $<0.01$ \\
Hypoglycaemia $(<4 \mathrm{mmol} / \mathrm{l})$ & $7 \pm 9$ & $6 \pm 8$ & 0.77 \\
Hyperglycaemia $(>12 \mathrm{mmol} / \mathrm{l})$ & $39 \pm 25$ & $27 \pm 18$ & $<0.05$ \\
CONGA & & & $<0.05$ \\
CONGA1 & $2.82 \pm 0.64$ & $2.58 \pm 0.80$ & 0.07 \\
CONGA2 & $4.13 \pm 1.0$ & $3.76 \pm 1.25$ & $<0.05$ \\
CONGA4 & $5.30 \pm 1.30$ & $4.75 \pm 1.56$ & \\
\hline
\end{tabular}


We observed, as expected, an improvement in $\mathrm{HbA}_{1 \mathrm{c}}$ after CSII commencement, although the period of 6-8 weeks would not allow the changes to be fully reflected by $\mathrm{HbA}_{1 \mathrm{c}}$. Blood glucose control was improved, as evidenced by greater time spent in the normoglycaemic range and less time in the hyperglycaemic range at follow-up as measured by CGMS. Blood glucose variability was also reduced as measured by a reduction in CONGA values calculated from CGMS. However, we were unable to demonstrate a direct relationship between improvement in glycaemic variables and improvement in behaviour and cognitive variables. We recognise that these findings are limited by having only performed $72 \mathrm{~h}$ of CGMS monitoring at each time-point and these may not accurately reflect longer periods. There was no difference in mean BGL at the time of cognitive testing across the two assessments, suggesting that any differences in performance were not attributable to variation in intercurrent BGLs.

While CSII patients were the group studied here, it may also be possible that similar effects could be found in groups of patients having intensification of therapy and significantly improving their control by changes in injection regimen. However, emerging data suggest advantages of CSII over injection regimens in terms of improving overall control and reducing BGL variability. It is also possible that improvements in mood and cognition could have been related to a number of other factors associated with starting an insulin pump, such as optimism and improved perception associated with a new technology, renewed motivation or a positive psychological response to increased diabetes input. While these factors are difficult to isolate, the advantages of improved confidence and perception should not be discounted, although longer term studies would be necessary to see if they are sustained.

Our findings relating to cognition suggest that after the commencement of insulin pump therapy children do not demonstrate improvement for tasks drawing on simple, information processing skills; however, moderate improvement is observed on tasks that are more complex and cognitively demanding and require more active mental processing, such as those involving cognitive flexibility and divided attention. These findings are consistent with existing neuropsychological literature, which suggests that, following subtle changes in brain function, deficits are less likely on tasks requiring simple, automatic responses and more likely on complex tasks requiring higher order cognitive skills [24]. We recognise that this is an uncontrolled pilot study; however, the cognitive measures used are not subject to significant practise effects according to available data $[17,18]$.

There are some published data demonstrating adverse effects of hypoglycaemia or hyperglycaemia on mental functioning, although the degree of blood glucose fluctua- tion required to affect these processes adversely is understood poorly. Deficits in cognitive functioning associated with hypoglycaemia have been reported to persist beyond the time of resolution of hypoglycaemia $[25,26]$ and one speculation is that hypoglycaemia may contribute to alterations in cerebral blood flow away from the dominant hemisphere [27]. It has also been postulated that recurrent episodes of hypoglycaemia may have a cumulative, detrimental effect on cognitive functioning [7, 8, 28] and poorer attention and organisational skills have been reported in those children whose diabetes began at a younger age [7, 29-31]. Regarding hyperglycaemia and cognitive functioning, Davis et al. showed that eight of 12 children with type 1 diabetes had a decrease in performance IQ (mean deterioration 6 IQ points) when hyperglycaemic (20-30 mmol/l) [19].

The improvements we observed in mood with commencement of CSII were consistent across parent, teacher and self-reports. In contrast, only parent reports indicated a significant improvement in behaviour, and this could relate to positive expectations as discussed above. However, these data are consistent with recent findings by McDonnell and colleagues [5], who found that more time spent in the hyperglycaemic range was associated with more externalising behaviours, which are more likely to be recognised by parents at home. Nocturnal hypoglycaemia has been shown to adversely affect mood in children the following day [28] and higher rates of mood variability have been documented in children with type 1 diabetes compared with sibling and classmate controls [32].

Our data suggest an association between improved glycaemic control after CSII commencement and improvements in more complex cognitive tasks, mood and behaviour. While we postulate that this might be caused by a reduction in blood glucose variability we were unable to demonstrate this relationship directly. Clearly, there may also be other positive factors associated with starting CSII that were not measured in this study. Nevertheless, these data give support to anecdotal parental and teacher observations that children's mood, behaviour and cognition improve following the commencement of insulin pump therapy. We hope that further study in a randomised controlled trial and longer follow-up will clarify these issues.

Acknowledgements This work was supported in part by a research grant and provision of equipment from Medtronic Australasia. Medtronic had no involvement in study design, conduct, data analysis or preparation and submission of the manuscript. The first draft of the manuscript was written by S. Knight. No form of payment was given to anyone to produce the manuscript.

Duality of interest The authors declare that there is no duality of interest associated with this manuscript. 


\section{References}

1. Brands AM, Biessels GJ, de Haan EH, Kappelle LJ, Kessels RP (2005) The effects of type 1 diabetes on cognitive performance: a meta-analysis. Diabetes Care 28:726-735

2. Desrocher M, Rovet J (2004) Neurocognitive correlates of type 1 diabetes mellitus in childhood. Child Neuropsychol 10:36-52

3. Northam EA, Rankins D, Cameron FJ (2006) Therapy insight: the impact of type 1 diabetes on brain development and function. Nat Clin Pract Neurol 2:78-86

4. Rankins D, Wellard RM, Cameron F, McDonnell C, Northam E (2005) The impact of acute hypoglycemia on neuropsychological and neurometabolite profiles in children with type 1 diabetes. Diabetes Care 28:2771-2773

5. McDonnell CM, Northam EA, Donath SM, Werther GA, Cameron FJ (2007) Hyperglycemia and externalizing behavior in children with type 1 diabetes. Diabetes Care 30:2211-2215

6. Rankins D, Wellard M, Cameron FJ, McDonnell CM, Northam EA (2005) The impact of acute hypoglycemia on neuropsychological and neurometabolite profiles in children with type 1 diabetes. Diabetes Care 28:2771-2773

7. Northam EA, Anderson PJ, Jacobs R, Hughes M, Warne GL, Werther GA (2001) Neuropsychological profiles of children with type 1 diabetes 6 years after disease onset. Diabetes Care 24:1541-1546

8. Northam EA, Anderson PJ, Werther GA, Warne GL, Adler RG, Andrewes D (1998) Neuropsychological complications of IDDM in children 2 years after disease onset. Diabetes Care 21:379-384

9. Cohen D, Weintrob N, Benzaquen H, Galatzer A, Fayman G, Phillip M (2003) Continuous subcutaneous insulin infusion versus multiple daily injections in adolescents with type I diabetes mellitus: a randomized open crossover trial. J Pediatr Endocrinol Metab 16:1047-1050

10. Doyle EA, Weinzimer SA, Steffen AT, Ahern JA, Vincent M, Tamborlane WV (2004) A randomized, prospective trial comparing the efficacy of continuous subcutaneous insulin infusion with multiple daily injections using insulin glargine. Diabetes Care 27:1554-1558

11. Kamoi K, Miyakoshi M, Maruyama R (2004) A quality-of-life assessment of intensive insulin therapy using insulin lispro switched from short-acting insulin and measured by an ITR-QOL questionnaire: a prospective comparison of multiple daily insulin injections and continuous subcutaneous insulin infusion. Diabetes Res Clin Pract 64:19-25

12. Linkeschova R, Raoul M, Bott U, Berger M, Spraul M (2002) Less severe hypoglycaemia, better metabolic control, and improved quality of life in type 1 diabetes mellitus with continuous subcutaneous insulin infusion (CSII) therapy; an observational study of 100 consecutive patients followed for a mean of 2 years. Diabet Med 19:746-751

13. McMahon SK, Airey FL, Marangou DA et al (2005) Insulin pump therapy in children and adolescents: improvements in key parameters of diabetes management including quality of life. Diabet Med 22:92-96
14. Daniel A (1983) Australian and New Zealand standard classification of occupations, 1st edn. Longman Cheshire, Melbourne

15. Anderson C, Lajoie G, Bell R (1995) Neuropsychological assessment of the school aged child. Melbourne University Press, Melbourne

16. Lezak M (1995) Neuropsychological assessment. Oxford University Press, New York

17. Wechsler D (2003) Wechsler intelligence for children - technical and interpretive manual. Harcourt Assessment, San Antonio, TX

18. Wechsler D (1999) Manual for the Wechsler abbreviated scale of intelligence. The Psychological Corporation, San Antonio, TX

19. Davis EA, Soong SA, Byrne GC, Jones TW (1996) Acute hyperglycaemia impairs cognitive function in children with IDDM. J Pediatr Endocrinol Metab 9:455-461

20. Holmes CS, Hayford JT, Gonzalez JL, Weydert JA (1983) A survey of cognitive functioning at difference glucose levels in diabetic persons. Diabetes Care 6:180-185

21. Draelos MT, Jacobson AM, Weinger K et al (1995) Cognitive function in patients with insulin-dependent diabetes mellitus during hyperglycemia and hypoglycemia. Am J Med 98:135-144

22. McDonnell C, Donath SM, Vidmar SI, Werther GA, Cameron FJ (2005) A novel approach to continuous glucose analysis utilising glycaemic variation. Diabetes Technol Ther 7:253-263

23. Daley KB, Wodrich DL, Hasan K (2006) Classroom attention in children with type 1 diabetes mellitus: the effect of stabilizing serum glucose. J Pediatr 148:201-206

24. Anderson V, Hendy J, Northam E, Wrennal J (2001) Developmental neuropsychology: a clinical approach. Psychology Press, London

25. Ryan CM, Atchison J, Puczynski S, Puczynski M, Arslanian S, Becker D (1990) Mild hypoglycemia associated with deterioration of mental efficiency in children with insulin-dependent diabetes mellitus. J Pediatr 117:32-38

26. Puczynski MS, Puczynski SS, Reich J, Kaspar JC, Emanuele MA (1990) Mental efficiency and hypoglycemia. J Dev Behav Pediatr $11: 170-174$

27. Tupola S, Salonen I, Hannonen R, Verho S, Saar P, Riikonen R (2004) Comparison of regional cerebral perfusion, EEG and cognitive functions in type 1 diabetic children with and without severe hypoglycaemia. Eur J Pediatr 163:335-336

28. Matyka KA, Wigg L, Pramming S, Stores G, Dunger DB (1999) Cognitive function and mood after profound nocturnal hypoglycaemia in prepubertal children with conventional insulin treatment for diabetes. Arch Dis Child 81:138-142

29. Hagen JW, Barclay CR, Anderson BJ et al (1990) Intellective functioning and strategy use in children with insulin-dependent diabetes mellitus. Child Dev 61:1714-1727

30. Rovet JF, Ehrlich RM, Hoppe M (1987) Intellectual deficits associated with early onset of insulin-dependent diabetes mellitus in children. Diabetes Care 10:510-515

31. Ryan C, Vega A, Drash A (1985) Cognitive deficits in adolescents who developed diabetes early in life. Pediatrics 75:921-927

32. McCarthy AM, Lindgren S, Mengeling MA, Tsalikian E, Engvall JC (2002) Effects of diabetes on learning in children. Pediatrics 109:e9 (Electronic article) 